\title{
ORIGINAL ARTICLE \\ The correlation between vertebral wedge-shaped changes in $X$-ray imaging at supine and standing positions and the efficacy of operative treatment of thoracolumbar spinal fracture in the elderly
}

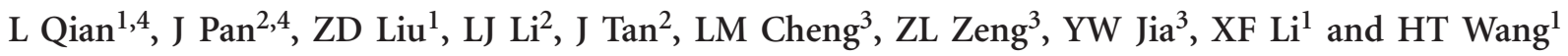

\begin{abstract}
Study design: By analyzing a large number of surgical patients, we identified the roles of wedge-shaped changes in related surgeries. Objectives: To illustrate the relevance of vertebral wedge-shaped changes in X-ray imaging at supine and standing positions in patients with percutaneous kyphoplasty as well as the postoperative effect.

Setting: All patient data were collected from a hospital in China.

Methods: Between June 2006 and May 2010, 77 surgical patients (9 men and 68 women) with wedge-shaped compression fractures were retrospectively analyzed. Patients were divided into group $A(\Delta W R \geqslant 2.5 \%)$ and group $B(\Delta W R<2.5 \%)$ according to the dynamic changes in the percentage of vertebral body wedge-shaped variable ratio (WR) at supine and standing positions. The intensity of back pain in different positions pre- and postoperatively was evaluated with a visual analog pain scale (VAS).

Results: The WRs in both standing and supine positions were significantly reduced by kyphoplasty in both groups A and B. In agreement with the improvement in WRs, the VAS was significantly decreased in three positions for patients in group A and in turning over and standing position for patients in group $B$. With respect to $\triangle W R$ changes, group $B$ revealed significantly lower values compared with group A preoperatively $(P<0.001)$, but there was no significant difference between groups $A$ and $B$ postoperatively and at 1-month follow-up ( $P=0.179$ and $P=0.558$, respectively).

Conclusions: Improvement in symptoms after kyphoplasty is better in patients with wedge-shaped changes in supine and standing positions, and the efficacy of height restoration of the spine would be better in unstable vertebrae by balloon dilatation.
\end{abstract}

Spinal Cord (2013) 51, 904-908; doi:10.1038/sc.2013.102; published online 17 September 2013

Keywords: wedge-shaped changes; supine and standing radiographs; kyphoplasty

\section{INTRODUCTION}

With the aging of the world's population, osteoporosis is becoming a prevalent public health problem worldwide. Osteoporosis-caused vertebral body compression fracture (VBCF) is one of the most common manifestations. In 2000, the number of osteoporotic fractures was estimated to be 3.79 million in the European Union and over 200 million worldwide. ${ }^{1}$ In the United States, there are almost 700000 new osteoporotic vertebral fractures, 250000 distal forearm fractures, 250000 hip fractures and 300000 fractures of other limb sites every year, one-third of which are associated with chronic pain. ${ }^{2}$ The annual incidence rate of VBCF in women over 50 years of age is higher than $1 \%$, whereas in women over 75 years of age it is three times higher. ${ }^{2,3}$ Short rests, less activity, brace fixation and non-steroidal medicine are extensively used to improve symptomatic fractures, ${ }^{4,5}$ whereas some patients, unsuited to conservative treatment, can undergo surgery. Currently, the main clinical diagnostic method for osteoporotic spinal fractures is Magnetic Resonance Imaging (MRI), ${ }^{6-8}$ and the proper determination of responsible vertebra is the key for success in surgical treatment. Some studies report changes in supine lateral and standing lateral radiographs in thoracolumar vertebral fractures 9,10 and the correlation of these changes with back pain. ${ }^{11}$ However, there is no report on the relationship between changes in vertebral height and surgical therapy effect. In this paper, a retrospective analysis was applied in patients undergoing percutaneous kyphoplasty to compare pre- and postoperative vertebral wedge-shaped changes in supine and standing positions and illustrate their relevance with operative effect.

\section{MATERIALS AND METHODS}

Between June 2006 and May 2010, 97 surgical patients (11 men and 86 women) with a simple thoracolumbar spinal compression fracture were admitted at our hospital. They were aged between 60 and 84 years, with a mean age of 70.7 years. All of them had a history of osteoporosis (T-score $\leqslant-2.5)$ before trauma and consulted a doctor for microtrauma or waist back pain without trauma, but none had neural symptoms of lower limb and other trauma. There are three types of osteoporotic fractures:

${ }^{1}$ Department of Orthopedic Surgery, Renji Hospital, Shanghai Jiaotong University School of Medicine, Shanghai, China; ${ }^{2}$ Department of Orthopedic Surgery, East Hospital of Tongji University, Shanghai, China and ${ }^{3}$ Department of Orthopedic Surgery, Tongji Hospital, Shanghai, China

${ }^{4}$ Co-first authors: Lie Qian, Jie Pan.

Correspondence: Dr L Qian or Z Liu, Department of orthopedic Surgery, Renji Hospital, Shanghai Jiaotong University School of Medicine, No. 1630 East Road, Shanghai 200127, China.

E-mail: lieqian123@hotmail.com

Received 2 March 2013; revised 22 July 2013; accepted 4 August 2013; published online 17 September 2013 
wedge-shaped change, crush or biconcave fractures. ${ }^{12}$ In this paper, only patients older than 60 years with a wedge-shaped compression fracture whose anterior compressed and posterior vertebrae were intact and with follow-up time of more than 1 month were included. A total of 20 patients with crush or biconcave fractures, as well as serious trauma, were excluded. The remaining 77 cases ( 9 men and 68 women) with wedge-shaped compression fracture and follow-up of 3 months were included. Fractures occurred at the level of T10 in 4 patients, T11 in 11 patients, T12 in 22 patients, L1 in 28 patients, L2 in 3 patients, L3 in 2 patients and at multiple levels in 7 patients. The time interval between pain occurrence and the procedure ranged from 1 month to 5 months (averaged of 40.2 days).

On MRI, the vertebral body that showed low signal intensity on T1weighted image (T1WI), high signal intensity on T2WI and high signal intensity in fat-suppression sequence served as the target. ${ }^{6}$ To measure anterior and posterior vertebral height of the injured segment vertebral body, lateral $\mathrm{X}$-ray radiographs were taken in supine and standing positions before and after surgery and at 1 month postoperatively. Four points of injured vertebrae in these X-ray radiographs were determined (Figure 1): Points (a) and (c) were placed at the most anterior-superior and anterior-inferior endplate margins, respectively. Points (b) and (d) were placed at the most posterior-superior and posterior-inferior endplate margins, respectively. Vertebral heights $(\mathrm{H}, \mathrm{mm})$ of anterior endplate $(\mathrm{Hb})$ and posterior endplate $(\mathrm{Ha})$ were determined as the distance between points $\mathrm{a}$ and $\mathrm{c}$ and distance between points $\mathrm{b}$ and $\mathrm{d}$, respectively. The percentage of vertebral body wedge-shaped variable ratio (WR) was calculated by using the formula $(\mathrm{Ha}-\mathrm{Hb}) / \mathrm{Ha} \times 100 \% .{ }^{11}$ Dynamic changes $(\Delta \mathrm{WR})$ in the wedge rate of supine and standing positions were calculated as WR (standing) - WR (supine). Patients were divided into groups A $(\Delta \mathrm{WR} \geqslant 2.5 \%)$ and $\mathrm{B}(\Delta \mathrm{WR}<2.5 \%)$ according to the changes in $\Delta \mathrm{WR}$ ( $\Delta \mathrm{WR}<2.5 \%$ mean no changes).

To evaluate the intensity of back pain, a $100-\mathrm{mm}$ visual analog pain scale (VAS) was applied to record pain intensity before and after surgery and at 1 month postoperatively. The intensity of back pain was recorded at supine

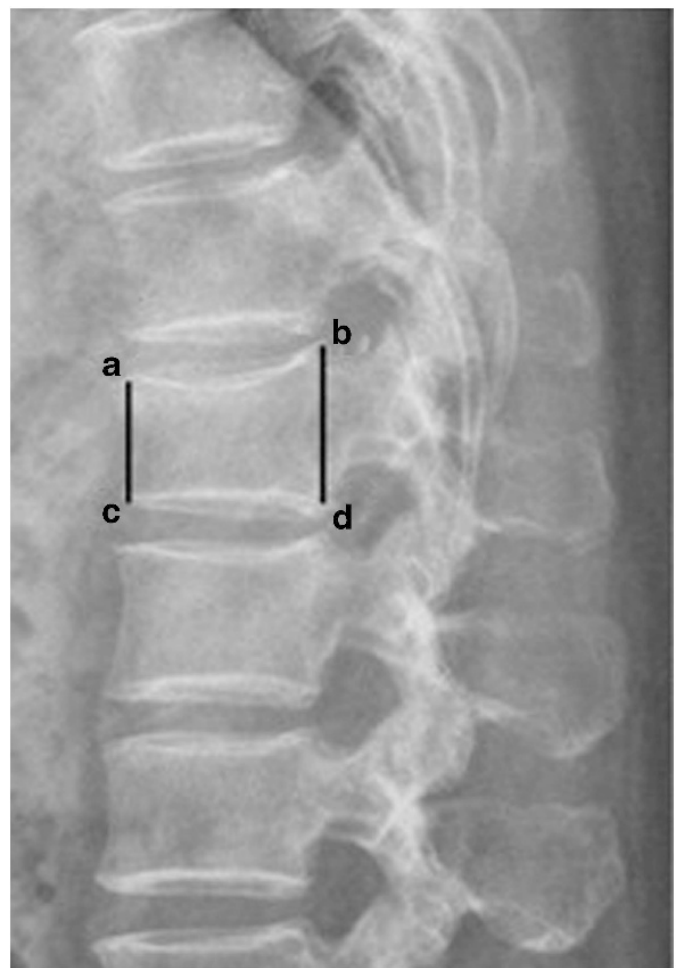

Figure 1 Four points (a-d) of injured vertebrae in X-ray radiographs were determined. Points (a) and (c) were placed at the most anterior-superior and anterior-inferior endplate margins, respectively. Points (b) and (d) were placed at the most posterior-superior and posterior-inferior endplate margins, respectively. position, turning over and standing position. In VAS, '0' represents no pain, whereas ' 100 ' represents the most serious pain. Pain alleviated rate $=$ (VAS (preoperative)_VAS (postoperative))/VAS (preoperative) $\times 100 \%$.

\section{Operation procedure}

Patients with an unstable vertebra or aggravated wedge-shaped changes were indicated for surgery. First, patients under general anesthesia were placed in the prone position. Under the fluoroscopic imaging guidance of a C-arm X-ray, a puncture point of vertebral pedicle in the injured vertebrae was determined and the projective skin was marked. After routine disinfection and after being covered with a piece of scarf, a $0.5-\mathrm{cm}$ cutaneous incision was made. Second, a bone biopsy needle was delivered into the first one-third of the vertebral body through the vertebral pedicle of the injured vertebra, and then the core of the puncture needle was removed and replaced with kirschner wire. An operation channel was established by separating tissues with a dilator and working cannula through the kirschner wire. Third, for the successive passage of the inflatable bone tamp (IBT), an intravertebral bone channel was created using a drill tip. Then the drill was removed and the IBT was inserted and pushed forward carefully and placed in the anterior two-thirds of the vertebra. The iohexol contrast medium was injected. An angioplasty injection device equipped with a pressure monitor was used to inflate the bone tamp. The end point of inflation was indicated by restoration of vertebral body height to normal height or attainment of the maximum volume of the balloon. Then bone cement was slowly applied to the cavity created by the IBT under X-ray fluoroscopy. At last, the cutaneous incisions were sutured and patients were instructed to remain in bed for the next few hours.

\section{Statistical analysis}

In the 77 patients, anterior to posterior vertebral height ratio was measured preoperatively and postoperatively to calculate the vertebral wedge-shaped changes. Pain was assessed based on the VAS before and after operation. All data were expressed as mean \pm s.d. and analyzed using the statistical software SPSS18.0 (Shanghai, China) using a paired $t$-test. $P<0.05$ was considered statistically significant.

\section{Statement of ethics}

We certify that all applicable institutional and governmental regulations concerning the ethical use of human volunteers were followed during the course of this research.

\section{RESULTS}

Surgeries were all successfully completed in the 77 patients with follow-up of more than 3 months (3-50 months, 18.2 months at average). Bone cement leakage occurred in four cases, without clinical compression symptom. The time to out-of-bed activity with waist protection was the second day after operation.

MRI examination in all patients before surgery showed low signal intensity on T1WI, high signal intensity on T2WI and high signal intensity in fat-suppression sequence. Patients were divided into group $\mathrm{A}(\Delta \mathrm{WR} \geqslant 2.5 \%)$ and group $\mathrm{B}(\Delta \mathrm{WR}<2.5 \%)$ according to their changes in $\Delta \mathrm{WR}$ of lateral $\mathrm{x}$-ray radiographs $(\Delta \mathrm{WR}<2.5 \%$ represents no changes). There were 61 and 16 cases in group A and group $\mathrm{B}$, respectively. The pre-procedure and post-procedure images in each group are shown in Figure $2(\Delta W R \geqslant 2.5 \%)$ and Figure 3 $(\Delta \mathrm{WR}<2.5 \%)$. The $\mathrm{WR}$ changes preoperatively, postoperatively and at postoperative follow-up (1 month) are shown in Table 1.

In group $\mathrm{A}$, the WR in standing position was significantly higher than that in supine position preoperatively $(P<0.001)$. The WRs in both standing and supine positions were significantly reduced by surgery, and there was no statistical significance between them ( $P=0.085$ after operation and $P=0.814$ at 1 -month follow-up). However, there was no significant difference between the WR in standing position and that in supine position preoperatively, 

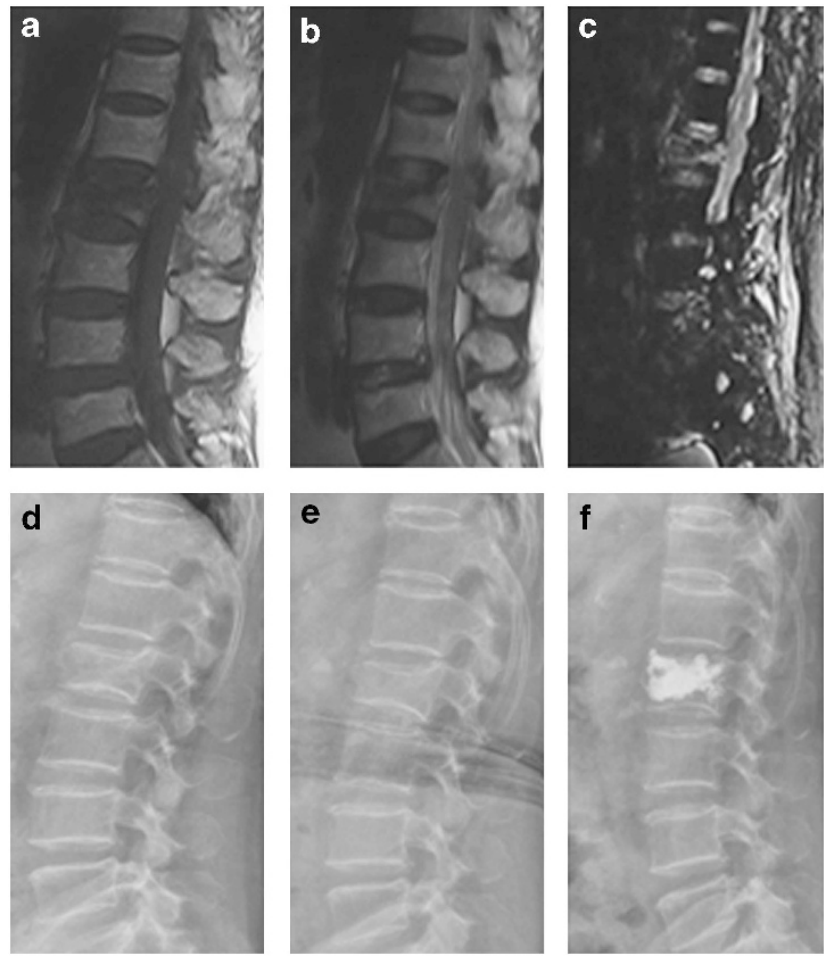

Figure 2 A 78-year-old woman sustained a compression fracture of L2 MRI showing low signal intensity at L2 on T1-weighted image (a) and T2-weighted image (b). MRI short time inversion recovery showing high signal intensity at L2 (c). Vertebral wedging rate (WR) of $19.76 \%$ in the supine position (d) increased to $36.61 \%$ on standing radiograph (e) and decreased to 6.02 (f) at 1 month postoperatively.

postoperatively and at 1-month follow-up $(P=0.944, P=0.402$ and $P=0.410$, respectively) in group $B$. Further, the statistical analysis between groups A and B indicated that the WR was significantly higher in group $\mathrm{B}$ at supine position postoperatively and at 1-month follow-up (all $P<0.001$ ), but there was no significant difference preoperatively between the two groups $(P=0.051)$. WR at standing position was significantly higher in group A than in group B preoperatively $(P=0.028)$, but significantly lower than in group $\mathrm{B}$ postoperatively and at follow-up (all $P<0.001$ ). As for $\Delta \mathrm{WR}$ changes, group $B$ was significantly lower than group A preoperatively $(P<0.001)$, but there was no significant difference between groups A and B postoperatively and at 1-month follow-up $(P=0.179$ and $P=0.558$, respectively).

\section{Pain assessment}

The intensity of back pain in different positions pre- and postoperatively was evaluated with VAS (Table 2). In group A, the VAS was significantly decreased after surgery in the supine position, turning over and standing position (all $P<0.001$ ) when compared with that before operation. The VAS was significantly decreased after operation in turning over and standing position $(P=0.003$ and $P<0.001$, respectively), but not in supine position $(P=0.188)$ in group $B$. Further, the statistical analysis between groups A and B indicated that the VAS was significantly lower in group B in turning over and standing position $(P<0.001)$, but there was no significant difference in supine position preoperatively $(P=0.626)$. In contrast, no significant difference was observed in supine position and turning over between groups $\mathrm{A}$ and $\mathrm{B}(P=0.247$ and $P=0.053$, respectively),
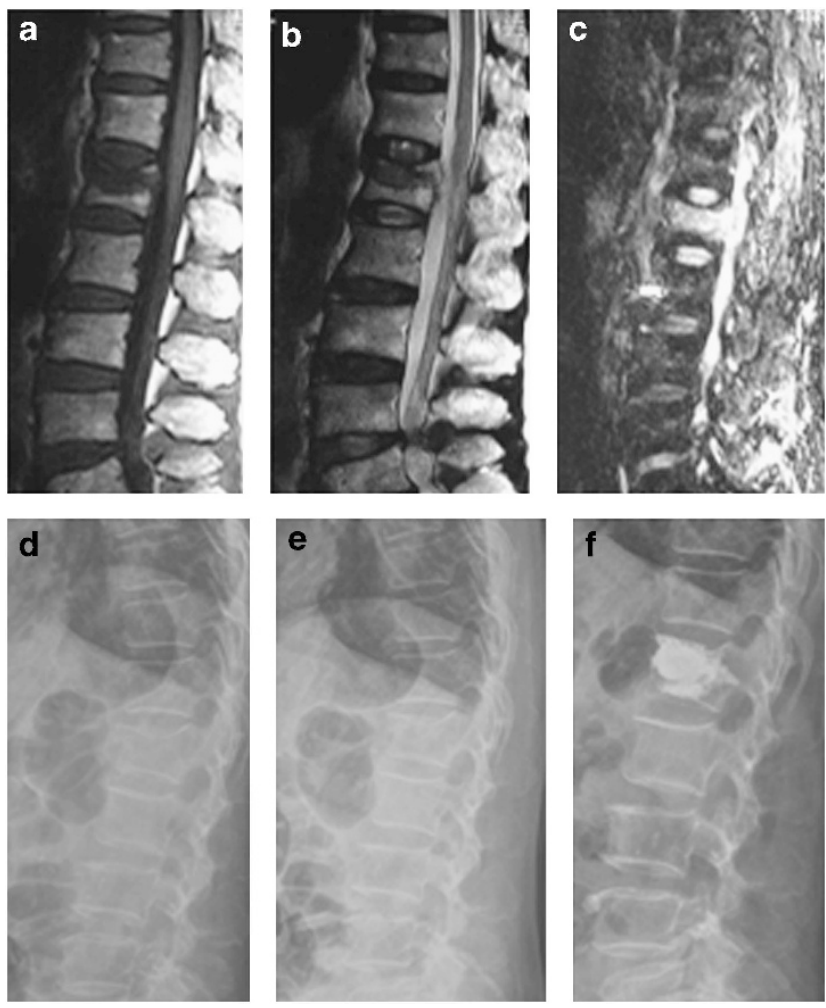

Figure 3 A 75-year-old woman sustained a compression fracture of L1. MRI showing low signal intensity at L1 on T1-weighted image (a) and T2-weighted image (b). MRI short time inversion recovery showing high signal intensity at L1 (c). Vertebral wedging rate (WR) at supine position (d) is $37.75 \%$, and that on standing radiograph (e) is $37.33 \%$. At 1 month postoperatively, the WR decreased to 11.67 (f).

whereas significant difference was observed in standing position postoperatively $(P=0.043)$. There were no significant differences between preoperative VAS and VAS at 1-month follow-up in any of the three positions in both groups A and B (all $P<0.001$ ). Overall, there was no significant difference between groups $A$ and $B$ in pain alleviated rate in supine position $(P=0.071)$, whereas there was significant difference in turning over and standing position (all $P<0.001)$.

\section{DISCUSSION}

Previously, nonoperative treatment was recommended for stable and burst VBCF. ${ }^{13}$ Although these treatments have been shown to be safe and effective, the patient's life quality was reduced because of lying in bed for a long time. Moreover, there are reports of complications of antiosteoporosis during these years. ${ }^{14}$ On the other hand, conventional open surgical treatment involves large dissections, prolonged anesthetic times and a high incidence of complications. In recent years, vertebroplasty/kyphoplasty has gained popularity gradually because of its minimally invasive feature, which reduces complications and improves life quality.

Since percutaneous vertebroplastywas reported by Galibert et al. ${ }^{15}$ in 1987 it has been widely used in treating vertebral tumors and VBCF. Although vertebroplasty is currently being used successfully for pain relief and vertebral reinforcement in $\mathrm{VBCF},{ }^{16}$ several disadvantages still exist, such as leakage of bone cement. ${ }^{17,18}$ In addition, this technique makes no attempt to restore the height of the collapsed vertebral body. In 2001, Lieberman et al. ${ }^{19}$ first performed 
Table 1 Wedge-shaped variable ratio

\begin{tabular}{|c|c|c|c|c|c|c|c|c|c|c|c|c|}
\hline \multirow[t]{2}{*}{ Group } & \multicolumn{4}{|c|}{ Preoperative } & \multicolumn{4}{|c|}{ Postoperative } & \multicolumn{4}{|c|}{ Follow-up (1 month postoperatively) } \\
\hline & WR (supine) & WR (standing) & $\Delta W R$ & P-value & WR (supine) & WR (standing) & $\Delta W R$ & P-value & WR (supine) & WR (standing) & $\Delta W R$ & P-value \\
\hline A & $28.52 \pm 6.69$ & $38.40 \pm 8.31$ & $9.88 \pm 5.40$ & $<0.001$ & $6.70 \pm 5.59$ & $6.97 \pm 5.65$ & $0.27 \pm 1.22$ & 0.085 & $7.02 \pm 5.53$ & $7.06 \pm 5.56$ & $0.04 \pm 1.30$ & 0.814 \\
\hline B & $33.04 \pm 8.04$ & $33.06 \pm 8.05$ & $0.03 \pm 1.39$ & 0.944 & $16.19 \pm 5.40$ & $15.81 \pm 5.01$ & $-0.38 \pm 1.77$ & 0.402 & $15.73 \pm 4.67$ & $15.96 \pm 5.32$ & $0.23 \pm 1.06$ & 0.410 \\
\hline$P$-value & 0.051 & 0.028 & $<0.001$ & & $<0.001$ & $<0.001$ & 0.179 & & $<0.001$ & $<0.001$ & 0.558 & \\
\hline
\end{tabular}

Data were analyzed by paired $t$-test and expressed as mean \pm s.d.

Table 2 Visual analog pain scale

\begin{tabular}{|c|c|c|c|c|c|c|c|c|c|c|c|}
\hline \multirow[t]{2}{*}{ Group } & \multicolumn{3}{|c|}{ Preoperative } & \multicolumn{4}{|c|}{ Postoperative } & \multicolumn{4}{|c|}{ Follow-up (1 month postoperatively) } \\
\hline & $\begin{array}{l}\text { Supine } \\
\text { position }\end{array}$ & Turning over & $\begin{array}{l}\text { Standing } \\
\text { position }\end{array}$ & $\begin{array}{l}\text { Supine } \\
\text { position }\end{array}$ & Turning over & $\begin{array}{l}\text { Standing } \\
\text { position }\end{array}$ & P-value & $\begin{array}{l}\text { Supine } \\
\text { position }\end{array}$ & Turning over & $\begin{array}{l}\text { Standing } \\
\text { position }\end{array}$ & P-value \\
\hline A & $8.69 \pm 8.66$ & $37.70 \pm 11.75$ & $49.84 \pm 13.84$ & $3.61 \pm 4.84$ & $5.25 \pm 5.66$ & $7.87 \pm 7.10$ & $\begin{array}{c}<0.001 \\
0.188\end{array}$ & $0.33 \pm 1.80$ & $0.98 \pm 3.00$ & $1.64 \pm 3.73$ & $<0.001$ \\
\hline B & $7.50 \pm 8.56$ & $18.75 \pm 8.85$ & $24.38 \pm 8.14$ & $5.63 \pm 6.29$ & $10.63 \pm 9.98$ & $13.75 \pm 10.25$ & $\begin{array}{c}0.003 \\
<0.001\end{array}$ & $1.88 \pm 4.03$ & $3.13 \pm 4.79$ & $5.63 \pm 8.14$ & $<0.001$ \\
\hline$P$ & 0.626 & $<0.001$ & $<0.001$ & 0.247 & 0.053 & 0.043 & & 0.153 & 0.105 & 0.074 & \\
\hline
\end{tabular}

Data were analyzed by paired $t$-test and expressed as mean \pm s.d.

percutaneous kyphoplasty by inserting an IBT into the vertebral body. VBCF was efficaciously treated with clinical improvement of pain and function as well as with restoration of the vertebral body.

Injured vertebra should be confirmed before kyphoplasty, and other diseases should also be ruled out, and hence it is key to identify the responsible vertebra among plenty of vertebrae with wedge changes. ${ }^{5}$ Careful physical examination is widely used in clinical and vertebral body with obvious spinous process tenderness and rap pain. MRI is then carried out to confirm the diagnosis on the basis of geographic patterns of low signal intensity on T1WI, high signal intensity on T2WI and high signal in fat-suppression sequence. Moreover, there is no correlation research to determine whether fracture vertebra with bleeding and edema is the main reason for pain. In clinics, kyphoplasty is usually carried out in more than one vertebra at a time because of uncertain responsible vertebrae, thus resulting in increased financial burden and surgical risk to the patient. Besides, there are potential cardiovascular complications and fat embolism. ${ }^{20}$ Moreover, many reports agree with the fact that fracture risk of adjacent segment vertebra increases after vertebroplasty. ${ }^{21-23}$ It has been reported that vertebroplasty should be performed for fewer than three vertebral bodies. ${ }^{24}$

Fracture instability due to biomechanical change is the main reason for pain in $\mathrm{VBCF},{ }^{25}$ and thus nonunion and unstable vertebrae are the therapeutic subjects. Only when bone cement fixes the fracture instability can satisfactory curative effect be achieved. The exact mechanism is unproven, but it may be because of the increased stabilization of the fractured vertebra and the elimination of macroscopic or microscopic motion at the fracture site. In addition, bone cement solidification can lead to thermogenesis, which will damage sensory nerve endings of the vertebral body. ${ }^{4}$ In this research, we judged the stability of the fracture vertebra according to the wedge-shaped changes shown in X-ray imaging in supine and standing positions, and evaluated the intensity of back pain in different positions pre- and postoperatively with VAS. Dynamic mobility was present when anterior vertebral height changed between standing and supine radiographs. There were three authors who reported the results of supine lateral and standing lateral radiographs in patients with VBCF. ${ }^{9-11}$ Among them, Toyone et al. ${ }^{11}$ suggested that there was a significant correlation between $\Delta \mathrm{WR}$ and back pain when standing erect. Results from our study showed that the WRs in both standing and supine positions were significantly reduced by kyphoplasty in both groups A and B (Table 1). In agreement with the improvement in WRs, VAS was significantly decreased in three positions for patients in group A and in turning over and standing position for patients in group B. This is consistent with the study by Toyone et al. ${ }^{11}$ With respect to $\Delta \mathrm{WR}$ changes, group B showed significantly lower values compared with group A preoperatively $(P<0.001)$, but there was no significant difference between groups $\mathrm{A}$ and $\mathrm{B}$ postoperatively and at 1-month follow-up $(P=0.179$ and $P=0.558$, respectively). These outcomes suggested that symptoms improvement after kyphoplasty was better in patients with significant wedge-shaped changes showing in X-ray imaging in supine and standing positions (group A). That is to say, the stability due to height restoration by balloon dilatation would be better in unstable vertebra, whereas for stable vertebra the height restoration was limited.

Aged patients with waist and back trauma often have accompanying soft-tissue injury. This symptom also appears in patients with VBCF. In this study, there was significant difference in VAS at different positions between patients in the two groups who underwent kyphoplasty, which indicated that there were other factors causing back pain, although the fractured vertebra was fixed and the pain caused by the fractured vertebra was alleviated. This suggested that patients with VBCF may also have soft-tissue injury. In addition, pain resulting from spinal fractures is due to muscle fatigue as well as due to arthritis in facet joints. ${ }^{4,26,27}$ Such pain can be alleviated in most patients by physical therapy and medical symptomatic treatment.

There are some limitations in our study. First, patients suffering from crush or biconcave fractures were not studied on account of the consideration of safety. Second, this is a retrospective study based on a 
relatively small number of cases, especially the number of cases in group B. Third, this study used only VAS for evaluating the effect of kyphoplasty. Other standard outcome measures for disability, such as Oswestry Disability Index, should be used in further studies.

\section{CONCLUSION}

Symptoms improvement after kyphoplasty is better in patients with significant wedge-shaped changes in supine and standing positions, and the efficacy of height restoration of spine by balloon dilatation would be better in unstable vertebrae. However, further prospective studies based on a larger number of cases with a long follow-up period are warranted to confirm our conclusion.

\section{DATA ARCHIVING}

There were no data to deposit.

\section{CONFLICT OF INTEREST}

The authors declare no conflict of interest.

1 Reginster JY, Burlet N. Osteoporosis: A still increasing prevalence. Bone 2006; 38 S4-S9.

2 Riggs BL, Melton LJ 3rd. The worldwide problem of osteoporosis: insights afforded by epidemiology. Bone 1995; 17: 505S-511S

3 Felsenberg D, Silman AJ, Lunt M, Armbrecht G, Ismail AA, Finn JD et al. Incidence of vertebral fracture in Europe: results from the European prospective osteoporosis study (epos). J Bone Miner Res 2002; 17: 716-724.

4 Rao RD, Singrakhia MD. Painful osteoporotic vertebral fracture. Pathogenesis, evaluation, and roles of vertebroplasty and kyphoplasty in its management. J Bone Joint Surg Am 2003; 85-A: 2010-2022.

5 Francis RM, Baillie SP, Chuck AJ, Crook PR, Dunn N, Fordham JN et al. Acute and long-term management of patients with vertebral fractures. QJM 2004; 97: 63-74.

6 Yamato M, Nishimura G, Kuramochi E, Saiki N, Fujioka M. Mr appearance at different ages of osteoporotic compression fractures of the vertebrae. Radiat Med 1998; 16: 329-334

7 Gu XH, Yang HL, Tang TS. Balloon kyphoplasty for aged multiple osteoporotic spinal fractures. Chinese J Bone Joint Injury 2005; 20: 151-153.

8 Dai LY. Mri in diagnosis of osteoporotic vertral fractures: Avascular necrosis of the vertebral body. J Clin Orthipaedics 2000; 3: 9-10.

9 Mehta JS, Reed MR, McVie JL, Sanderson PL. Weight-bearing radiographs in thoracolumbar fractures: Do they influence management? Spine 2004; 29: 564-567.
10 McKiernan F, Faciszewski T. Intravertebral clefts in osteoporotic vertebral compression fractures. Arthritis Rheum 2003; 48: 1414-1419.

11 Toyone T, Tanaka T, Wada Y, Kamikawa K, Ito M, Kimura K et al. Changes in vertebra wedging rate between supine and standing position and its association with back pain a prospective study in patients with osteoporotic vertebral compression fractures. Spine 2006; 31: 2963-2966.

12 Ismail AA, Cooper C, Felsenberg D, Varlow J, Kanis JA, Silman AJ et al. Number and type of vertebral deformities: Epidemiological characteristics and relation to back pain and height loss. European vertebral osteoporosis study group. Osteoporos Int 1999; 9 206-213.

13 Cantor JB, Lebwohl NH, Garvey T, Eismont FJ. Nonoperative management of stable thoracolumbar burst fractures with early ambulation and bracing. Spine 1993; 18 971-976.

14 Hoang-Kim A, Gelsomini L, Luciani D, Moroni A, Giannini S. Fracture healing and drug therapies in osteoporosis. Clin Cases Miner Bone Metab 2009; 6: 136-143.

15 Galibert P, Deramond H, Rosat P, Le Gars D. Preliminary note on the treatment of vertebral angioma by percutaneous acrylic vertebroplasty. Neurochirurgie 1987; 33: $166-168$.

16 Watts NB, Harris ST, Genant HK. Treatment of painful osteoporotic vertebra fractures with percutaneous vertebroplasty or kyphoplasty. Osteoporos Int 2001; 12 . 429-437.

17 Lai PL, Tai CL, Chen LH, Nien NY. Cement leakage causes potential thermal injury in vertebroplasty. BMC Musculoskelet Disord 2011; 12: 116.

18 Teng MM, Cheng $\mathrm{H}$, Ho DM, Chang CY. Intraspinal leakage of bone cement after vertebroplasty: A report of 3 cases. AJNR Am J Neuroradiol 2006; 27: 224-229.

19 Lieberman IH, Dudeney S, Reinhardt MK, Bell G. Initial outcome and efficacy of "kyphoplasty" in the treatment of painful osteoporotic vertebral compression fractures. Spine 2001: 26: 1631-1638.

20 Aebli N, Krebs J, Schwenke D, Davis G, Theis JC. Pressurization of vertebral bodies during vertebroplasty causes cardiovascular complications: An experimental study in sheep. Spine 2003; 28: 1513-1519.

21 Ahn Y, Lee JH, Lee HY, Lee SH, Keem SH. Predictive factors for subsequent vertebral fracture after percutaneous vertebroplasty. J Neurosurg Spine 2008; 9: 129-136.

22 Lin EP, Ekholm S, Hiwatashi A, Westesson PL. Vertebroplasty: Cement leakage into the disc increases the risk of new fracture of adjacent vertebral body. AJNR Am J Neuroradiol 2004; 25: 175-180.

23 Trout AT, Kallmes DF, Kaufmann TJ. New fractures after vertebroplasty: Adjacent fractures occur significantly sooner. AJNR Am J Neuroradiol 2006; 27: 217-223.

24 Mathis JM, Barr JD, Belkoff SM, Barr MS, Jensen ME, Deramond H. Percutaneous vertebroplasty: A developing standard of care for vertebral compression fractures. AJNR Am J Neuroradiol 2001; 22: 373-381.

25 Barr JD, Barr MS, Lemley TJ, McCann RM. Percutaneous vertebroplasty for pain relief and spinal stabilization. Spine 2000; 25: 923-928.

26 Oleksik A, Lips P, Dawson A, Minshall ME, Shen W, Cooper C et al. Health-related quality of life in postmenopausal women with low bmd with or without prevalent vertebral fractures. J Bone Miner Res 2000; 15: 1384-1392.

27 Huang C, Ross PD, Wasnich RD. Vertebral fracture and other predictors of physica impairment and health care utilization. Arch Intern Med 1996; 156: 2469-2475. 